\title{
Arbitrarily shaped Point Spread Function (PSF) estimation for single image blind deblurring
}

DOI:

10.1007/s00371-020-01930-5

\section{Document Version}

Accepted author manuscript

Link to publication record in Manchester Research Explorer

\section{Citation for published version (APA):}

Khan, A., \& Yin, H. (2020). Arbitrarily shaped Point Spread Function (PSF) estimation for single image blind deblurring. Visual Computer. https://doi.org/10.1007/s00371-020-01930-5

\section{Published in:}

Visual Computer

\section{Citing this paper}

Please note that where the full-text provided on Manchester Research Explorer is the Author Accepted Manuscript or Proof version this may differ from the final Published version. If citing, it is advised that you check and use the publisher's definitive version.

\section{General rights}

Copyright and moral rights for the publications made accessible in the Research Explorer are retained by the authors and/or other copyright owners and it is a condition of accessing publications that users recognise and abide by the legal requirements associated with these rights.

\section{Takedown policy}

If you believe that this document breaches copyright please refer to the University of Manchester's Takedown Procedures [http://man.ac.uk/04Y6Bo] or contact uml.scholarlycommunications@manchester.ac.uk providing relevant details, so we can investigate your claim.

\section{OPEN ACCESS}




\title{
Arbitrarily Shaped Point Spread Function (PSF) Estimation for Single Image Blind Deblurring
}

\author{
Aftab Khan • Hujun Yin
}

Received: date / Accepted: date

\begin{abstract}
The research paper focuses on a challenging task faced in Blind Image Deblurring (BID). It relates to the estimation of arbitrarily shaped (non-parametric or complex shaped) Point Spread Functions (PSFs) of motion blur caused by camera handshake. These PSFs exhibit much more complex shapes than their parametric counterparts and deblurring, in this case, requires intricate ways to estimate the blur and effectively remove it. This research work introduces a novel blind deblurring scheme visualized for deblurring images corrupted by arbitrarily shaped PSFs. It is based on Genetic Algorithm (GA) and utilizes the Blind/Reference-less Image Spatial QUality Evaluator (BRISQUE) measure as the fitness function for arbitrarily shaped PSF estimation. The proposed BID scheme has been compared with other state-of-the-art single image motion deblurring schemes as benchmarks. Validation has been carried out on the standard real-life blurred images. Results of both benchmark and real images are presented. For real-life blurred images, the proposed BID scheme using BRISQUE converges in close vicinity of the original blurring functions. However, the benchmark schemes fail to effectively restore the real blurred images. The proposed scheme surpasses on average of seven percent higher image quality as compared to the benchmark schemes.
\end{abstract}

\section{Aftab Khan}

Department of Computer Systems Engineering. University of Engineering and Technology (UET), Peshawar, Pakistan. 25120

Tel.: +92-91-9222233

E-mail: aftab.khan@uetpeshawar.edu.pk

Hujun Yin

School of Electrical and Electronic Engineering, The University of Manchester, UK. M13 9PL
Keywords Blind Image Deblurring (BID) · Genetic Algorithm · Image Restoration · Image Quality Measures · Filter Estimation · Deconvolution.

\section{Introduction}

The PSF for uniform blurring can be estimated using the functional form for some common types of blur i.e. the Gaussian blur, motion blur and out-of-focus blur [1]. In real life, especially in the case of motion blur resulting from camera handshake, the blur follows convoluted paths resulting in complex-shaped PSFs [2,3]. There exist many schemes in the literature dealing with the restoration of such images with some listed in [3-12]. The blurring PSF shape, in this case, cannot be easily modelled by a simple equation or defined by a mathematical model for a set of its parameter(s) [2]. PSFs in such cases exhibit an arbitrary shape and deblurring, in this case, requires intricate ways to estimate the blur and effectively remove it. Such arbitrarily shaped PSFs have been shown to exist in the case of atmospheric turbulence blur as well as [13].

Some examples of real blurred images corrupted by arbitrarily shaped PSFs are shown in Fig. 1(a), with their respective close-ups in Fig. 1(b). The camera shake pictures exhibit PSFs far different from that found when the linear motion was investigated in [14]. The handsketched PSFs are shown in Fig. 1(c). Though modelling or approximating such PSFs using a large set of parameters is not impossible, estimating in such case becomes challenging, both computationally and in terms of convergence of the BID algorithm.

This research work focuses on the challenging task of restoring of images blurred by arbitrarily shaped PSF resulting from motion blur due to camera handshake. 
(a)

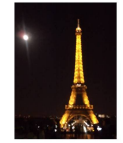

(b)

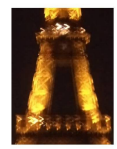

(c)

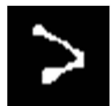

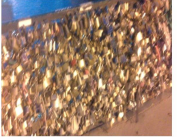
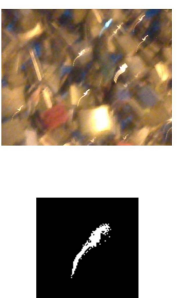
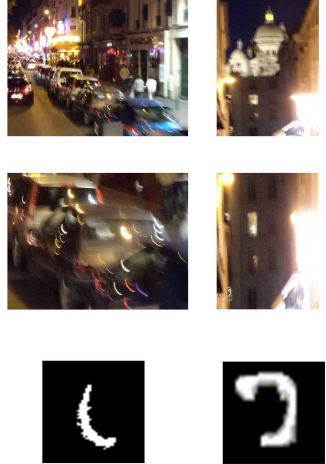

Fig. 1 Examples of arbitrarily shaped PSFs. Row 1. Real blurred images. Row 2. Image sections and Row 3. Corresponding hand sketched PSFs.

It uses a blind Image Quality Measure (IQM) as feedback of deblurred image quality. Blind/Reference-less Image Spatial QUality Evaluator (BRISQUE) IQM is used as a fitness function to the Genetic Algorithm (GA).However, for real-life blurred images, RPSNR IQM is a more robust substitute as it is less affected by deblurring noise and ringing. The coefficients of the 2D PSF are represented by binary chromosomes which evolve for the optimum values of the IQM hence optimizing the search for the blurring PSF.

The remainder of the paper is organized as follows. The next section briefly introduces some of the blind IQMs used for evaluating the image quality without a reference high-quality image. Section II describes the proposed learning and blind deconvolution scheme in detail while Section IV presents the details of the PSF support size estimation technique. Experimental setup and deblurring results for artificially blurred and real blurred images suffered from various forms of degradation and their analysis are given in Section 4,5, and 6 respectively. Discussion and analysis of the whole research work are given in Section 7 whereas concluding remarks are presented in Section 8.

The next section details the proposed BID scheme for arbitrarily shaped PSF estimation and deblurring.

\section{Proposed Blind Deblurring Scheme For Arbitrarily Shaped PSF Estimation}

The main idea behind the proposed BID scheme is stated as follows:

Any parametric or non-parametric uniform PSF can be approximated by estimating its coefficients values using a deblurring measure as feedback of deblurred image quality to the BID scheme.

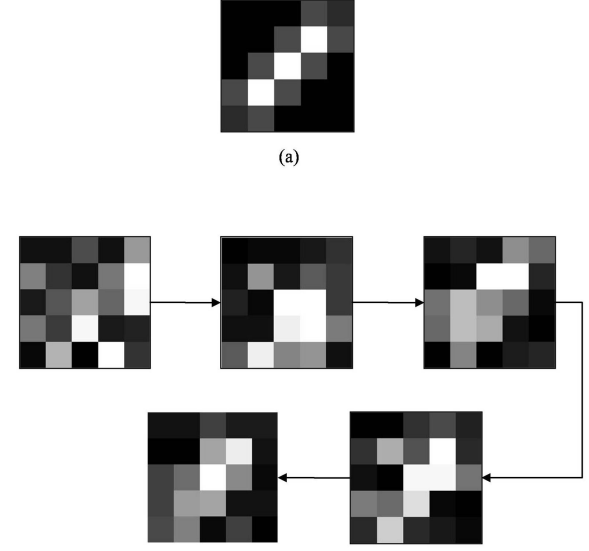

(b)

Fig. 2 (a) Blurring PSF (b) An overview of the arbitrarily shaped PSF estimation process through different stages.

A PSF can be assumed as a matrix of random values which can be estimated under a set of constraints. Mentioned below are the constraints applied to the PSF matrix in this research work:

- The PSF has a finite support size, with a finite number of rows and columns $m$ and $n$, respectively.

- The energy of the PSF is maintained. i.e. $\sum_{m, n} h(m, n)=1$

- The PSF is space invariant. The same blurring effect is presented by the blurring kernel at each pixel location.

- The PSF coefficients are non-negative.

During each iteration, a coefficient value is updated in the direction of improved deblurred image quality. The deblurred image quality can be calculated using an Image Quality Measure (IQM). The IQM can lead the BID scheme towards blurring PSF estimation and in turn restoring the blurred image. The process can be evaluated for a fixed number of iterations or it can be terminated when the difference in the IQM's value in subsequent iterations is lower than a specified threshold value.

Fig. 2 shows a glimpse of the restoration process for estimating the PSF coefficients. The original PSF is depicted in Fig. 2 (a) while the estimation process is illustrated in Fig. 2 (b). From an initial set of random values, the process keeps on estimating the PSF coefficients' weights till the IQM stops showing any further improvement.

An added advantage of such a BID scheme is that the same method can easily be extended for estimating other types of blur apart from camera handshake. It can be used to estimate parametric and non-parametric blur of different types. 
The deblurred image quality can be calculated using any blind IQM such as the non-Gaussianity measures spatial kurtosis [21] and spectral kurtosis [18] and other blind IQMs including Reblurring based Peak Signal to Noise Ratio (RPSNR) [22], BRISQUE [19,23] and NIQE $[20,24]$ to name a few.

The deblurring scheme is optimized using GA with IQM as the fitness function. The ASP-BID scheme based on GA is flexible as it can be easily incorporated with any IQM as the fitness function. Details of the GA based search optimization for the proposed BID scheme are as follows:

- Step 1: Initialize the GA parameters i.e. population, size, crossover rate, mutation rate etc.

- Step 2: Generate an initial chromosome population where each chromosome contains information about all the coefficients of the finite support size PSF.

- Step 3: Perform iteration and find the restored image through Wiener filtering for all the chromosomes.

- Step 4: Calculate fitness function values for the initial population.

- Step 5: Select the best fitting group of chromosomes based on either roulette or threshold-based selection.

- Step 6: Generate a new population from the chromosomes selected in Step 5 through crossover and mutation. Each crossover is performed with probability $p_{c}$ in the range of $0.5,0.8$ and cross over points are selected at random. Mutation involves modification of components of the individual chromosomes with probability $p_{m} \cdot p_{m}$ is usually a small number usually assumed in the range of $0.001, \ldots . ., 0.01$. Roulette wheel selection is used to select the best fitting individuals among the population.

- Step 7: Repeat the process again from Step 3 until the algorithm converges for the deblurring measure.

The proposed BID scheme requires PSF size input from the user so a finite matrix of coefficient values is calculated. In this regard, a method for visual judgement based PSF size estimation before deblurring is discussed in Section IV. In the case of real blurred images, the PSF size was estimated by inspecting a uniform blur region in the blurred image.

\section{PSF Support Size Estimation}

In order to estimate the PSF support size, a simple technique is proposed here. Initially, the GA is run for different sizes of PSF for a couple of iterations. The results are then visually judged for ringing artefacts.
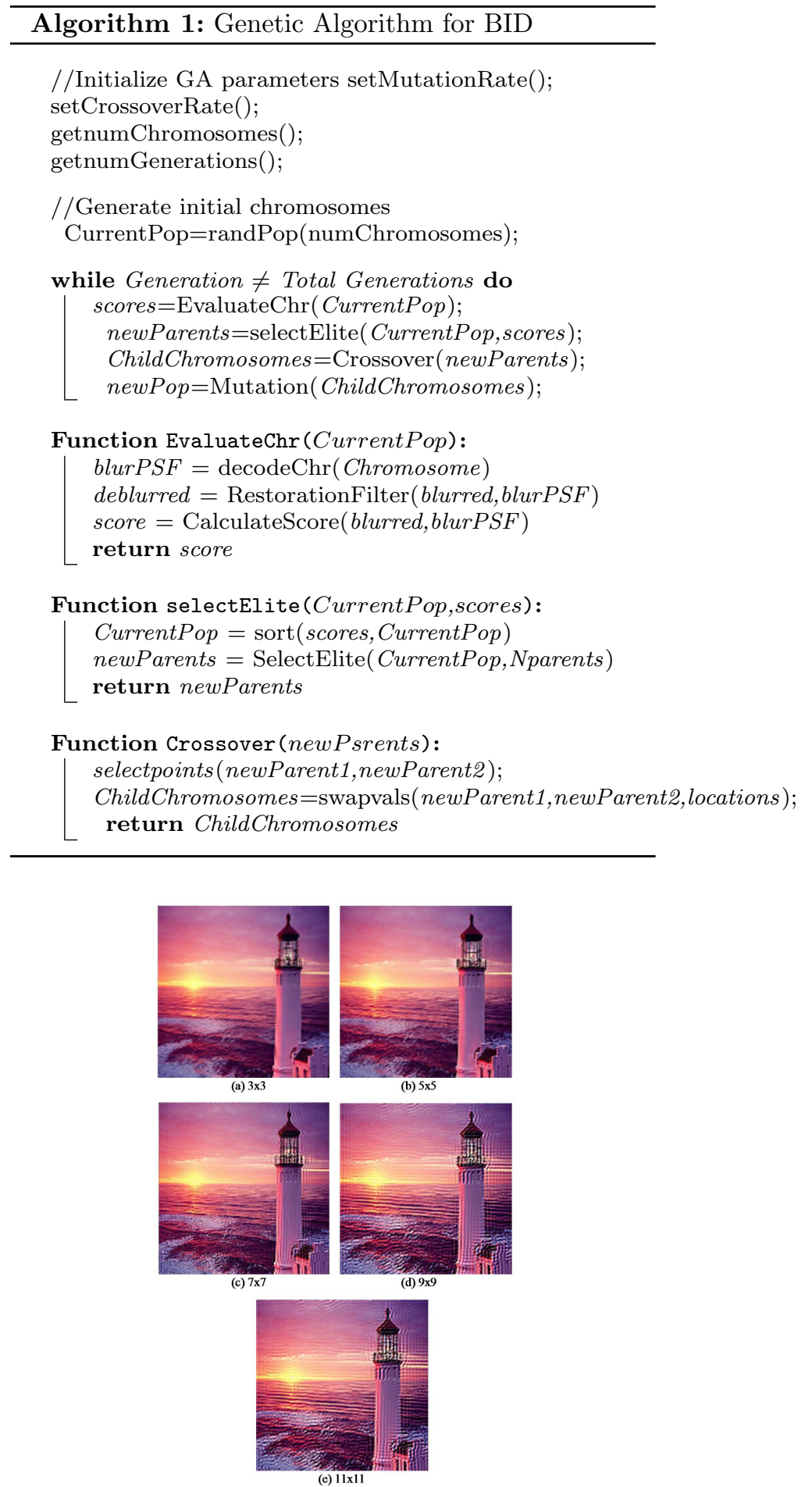

Fig. 3 Deblurring results for varying PSF sizes. A smaller PSF coefficient matrix results in almost no deblurring, while ringing artifacts are observed PSF sizes larger than 5x5. 


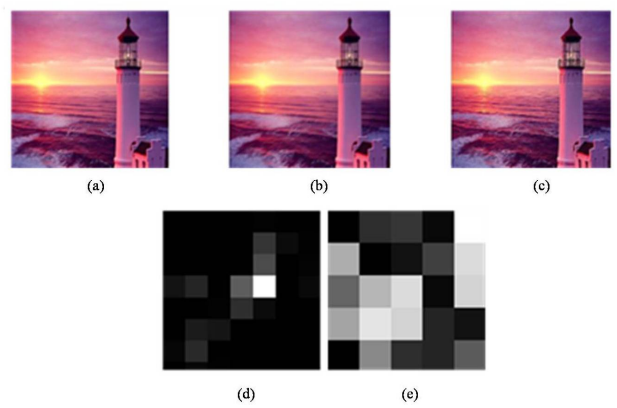

Fig. 4 Deblurring result for arbitrarily shaped PSF of size $5 \times 5$ pixels estimated by visual judgment of ringing artifacts. (a) Original image (b) Blurred image (c) Deblurred image (d) Blurring PSF and (e) Estimated PSF of size $5 \times 5$ pixels.

Initially, if the estimated PSF is bigger in size than the blurring PSF, it creates estimation error among them. This results in ringing artefacts visible in the deblurred image. The more the PSF estimation error, the severe the ringing artefacts in the deblurred image. If the PSF estimation error is minimum, ringing is least. The user can thus pick a PSF size which shows reduced ringing for the deblurred image.

Fig. 3 shows the image deblurred for varying sizes of PSF. The initial blur PSF size was an $8 \times 7$ coefficient matrix. The $5 \times 5$ matrix PSF depicts image sharpness as compared to the $3 \times 3$ matrix. All other bigger size matrices result in severe ringing in the deblurred image. The deblurring result for the $5 \times 5$ size estimated PSF is shown in Fig. 4.

\section{Experimental Setup}

Test images include images from the Waterloo Bragzone grayscale image dataset [25] as well as a collection of real-life blurred images mostly captured by the first author himself. Sony DSC-W310 digital camera was used to capture the real blurred images. The real-life camera handshake images demonstrate a reasonable amount of image noise as well. Noise can affect the deblurring results by changing the statistical properties of the image. Initially, a variant of GA coded by ourselves was employed for optimizing the search. After MATLAB released support for GA in its optimization toolbox [26], the proposed BID was successfully implemented on it and evaluated in parallel on a multiple core computer. Optimization of the proposed BID scheme is out of the scope of this research work thus further details of its execution on the multiple core machine have been omitted.

Six blind (non-reference) image quality measures were used. These quality measures are based on the $\mathrm{Hu}-$ man Visual System (HVS). The latest and robust non- reference (totally blind) image quality measures have been used to gauge the performance of the deblurring schemes. This includes BRISQUE, NIQE, BLIINDS, DIIVINE, FRIQUEE, SSEQ [19,20]. Spearman correlation coefficients of the IQM scores with Human scores from various subjects are also presented. For testing on the real-life blur images, 18 of our images and 100 images from the Lai dataset [27] were used.

The proposed ASP-BID schemes estimated PSF coefficients are not exactly the same as the original PSF but are rather a near approximation of the original blurring PSF. The proposed BID scheme estimates the PSF coefficients for a limited number of iterations which produces a reasonable approximation of the original PSF coefficients. This affects the brightness and contrast of the deblurred images thus rendering the full-reference IQMs: the Mean Structural Similarity (MSSIM) index [28-30] and the Universal Quality Index (UQI) [31] etc. invalid for quantitative image quality evaluation. Instead, non-reference IQMs BRISQUE and NIQE were used for this purpose.

BRISQUE was also used as the deblurring measure for the restoration of the images due to its efficient deblurring performance among multiple IQMs. The different IQMs compared for BID included spatial and spectral kurtosis, RPSNR, BRISQUE and NIQE IQM. The related work is detailed in [32]. Deblurring results for artificially blurred and real blurred images are presented below.

\section{Deblurring Results For Artificially Blurred Images}

Since the BID scheme can easily be extended for uniform parametric blurs, the algorithm was first evaluated for the less complex parametric form blurs before testing it for arbitrarily shaped PSFs. Deblurring results for Gaussian, motion and out-of-focus blur are presented below.

\subsection{Restoration of Parametric PSF Blurred Images}

The first set of tests included deblurring images blurred by Gaussian PSF. Fig. 5(a) shows the deblurring result for Cameraman image blurred by Gaussian PSF of size $11 \times 11$ pixels and variance $\sigma^{2}=2$. It can be observed that the estimated PSF takes on a rough shape of the blurring PSF as viewed in Fig. 5(a). Results presented here were obtained when the algorithm stopped, as the tolerance value for the fitness function was attained. The restored image appears sharper and much more detailed than its blurred counterpart. 
(a)

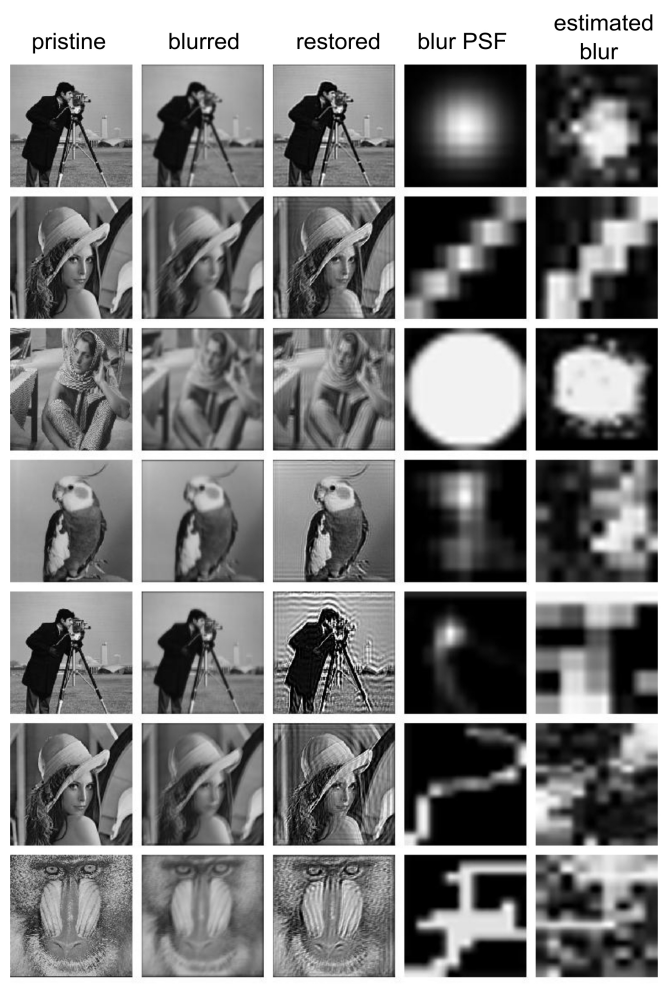

Fig. 5 Deblurring result for image blurred by Gaussian PSF of size $11 \times 11$ pixels and variance $s i g m a^{2}=2$. (a) Original image (b) Blurred image (c) Deblurred image (d) Blurring PSF and (e) Estimated PSF.

The second set of tests included deblurring images artificially blurred by motion blur. Fig. 5(b) shows the deblurring results for the Lena image blurred by motion blur PSF of length 11 pixels and angle 23 degrees. The estimated PSF achieved for the fixed iterations of the deblurring algorithm is almost similar to the blurring kernel.

Fig. 5(c) shows the deblurring result of the Barbara images under the influence of out-of-focus blur of radius 9 pixels. Deblurring results show the estimated PSF converging towards the original blur PSF. For the large out-of-focus blur, a lot of attenuation occurs for the high-frequency elements in the image and recovery, in this case, is not that sharp as observed for the Barbara image.

5.2 Deblurring Images Blurred by Arbitrarily Shaped PSFs

Arbitrarily shaped PSFs was used to blur the images and then recover using the proposed scheme. Deblurring results are shown in Fig. 5(d) - 5(g) which depict
Table 1 Deblurring results for artificially blurred images. Deblurred image quality is evaluated using BRISQUE and NIQE IQM.

\begin{tabular}{|c|c|c|c|}
\hline $\begin{array}{l}\text { Figure } \\
\text { Number }\end{array}$ & Filter & $\begin{array}{c}\text { Size } \\
\text { (pixels) }\end{array}$ & $\begin{array}{c}\text { Parameter } \\
\text { Values }\end{array}$ \\
\hline Fig. 5(a) & Gaussian & $11 * 11$ & sigma $^{2}=2.0$ \\
\hline Fig. 5(b) & Motion & $5 * 11$ & $\mathrm{~L}=11, \mathrm{~A}=23$ \\
\hline Fig. 5(c) & Out-of-focus & $19 * 19$ & $\mathrm{R}=9$ \\
\hline Fig. 5(d) & Arbitrary & $9 * 9$ & NA \\
\hline Fig. 5(e) & Arbitrary & $15^{*} 11$ & NA \\
\hline Fig. 5(f) & Arbitrary & $16^{*} 10$ & NA \\
\hline \multirow[t]{3}{*}{ Fig. 5(g) } & Arbitrary & $11 * 14$ & NA \\
\hline & \multicolumn{3}{|c|}{ BRISQUE } \\
\hline & Pristine & Blurred & Deblurred \\
\hline Fig. 5(a) & 6.68 & 35.88 & 47.27 \\
\hline Fig. 5(b) & 10.26 & 16.67 & 52.07 \\
\hline Fig. 5(c) & 50.12 & 30.57 & 63.38 \\
\hline Fig. 5(d) & 14.30 & 14.67 & 39.11 \\
\hline Fig. 5(e) & 14.13 & 17.95 & 36.43 \\
\hline Fig. 5(f) & 15.45 & 20.77 & 49.80 \\
\hline \multirow[t]{3}{*}{ Fig. 5(g) } & 49.04 & 30.07 & 42.27 \\
\hline & \multicolumn{3}{|c|}{ NIQE } \\
\hline & Pristine & Blurred & Deblurred \\
\hline Fig. 5(a) & 5.11 & 4.57 & 6.89 \\
\hline Fig. 5(b) & 5.11 & 4.69 & 7.19 \\
\hline Fig. 5(c) & 4.98 & 6.12 & 9.66 \\
\hline Fig. 5(d) & 4.74 & 4.74 & 7.43 \\
\hline Fig. 5(e) & 5.50 & 5.94 & 7.56 \\
\hline Fig. 5(f) & 4.98 & 5.72 & 6.30 \\
\hline Fig. 5(g) & 6.42 & 8.85 & 7.94 \\
\hline
\end{tabular}

that the proposed algorithm was able to estimate the blurring coefficients to a large extent. The deblurred images appear sharper than their blurred counterparts.

In Fig. 5(e), it can be observed that the estimated PSF was of size $5 \times 5$ pixels while the actual blurring kernel was of size $15 \times 11$ pixels. The size for the estimated PSF was selected as $5 \times 5$ pixels because a bigger support size PSF resulted in increased ringing in the deblurred image. A $5 \times 5$ coefficient matrix turned out as the best choice for estimating the original PSFs coefficient values.

Table 1 illustrates the quantitative IQMs values for the deblurred images using the arbitrarily shaped PSF estimation scheme. BRISQUE and NIQE values for the original, blurred and deblurred image are shown as comparison of deblurred image quality.

Low values of BRISQUE and NIQE represent high quality images. For Fig. 5(g), both NIQE and BRISQUE show improvement in the image. It can be seen that the ringing in the deblurred image is minimal. 
Though the deblurred images in Fig. 5(a)-5(g) have recovered well from the blurring as observed visually, the BRISQUE and NIQE values erroneously depict the deblurred images are of less visual quality. This is due to the presence of residual blur left from incomplete deblurring using the PSF estimates that are not the same as the original blurring PSFs. The tests also show that BRISQUE and NIQE IQMs may not be ideal blind IQMs and more effort is still needed to enhance their performance.

\section{Restoration of Real Blurred Images}

Real-life blurred images were used to test the efficacy of the deblurring scheme for practical applications. The images depict motion blur resulting from camera handshake. The BID schemes of Fergus et al. [3] and Whyte et al. [5] were used as a benchmark for comparison of deblurred image quality. These schemes were picked out due to the availability of their code. Other schemes were omitted from comparison due to their code complexity and large computation time A brief discussion of these schemes is provided below.

The scheme from Fergus et al. estimates the blurring PSF and then deconvolves the image using a RichardsonLucy filter. The PSF estimation process depends on image statistics, especially pixel color/image gradients. Their scheme was designed to estimate in-plane motion PSF while neglecting out-of-plane (rotational) motion blur. The BID scheme by Whyte et al. extends the scheme of Fergus et al. by incorporating rotational blur constraints as well as employing it for two deblurring cases. In the first deblurring case their scheme has been used to deblur a single-shot image, while in the second deblurring case, it utilizes information from a noisy pair of the blurred image along with a single-shot blurred image to estimate the blurring PSF.

Fig 6 shows the deblurring results for the Ian-1 image with the image source given in [33]. The blurred image is shown in Fig. 6(a). The deblurred image using the proposed BID scheme is shown in Fig. 6(b) with the estimated PSF in Fig. 6(e). The blurred image after restoration is reasonably clear with some ringing in Fig. 6 (b) for the proposed scheme. However, the best result in terms of visual quality is achieved for the Fergus et al. based BID scheme in Fig. 6(c). Whyte et al.'s scheme was unable to estimate the blurring kernel properly and the deblurred image in Fig. 6(d) contains large residual blur and ringing.

Deblurring results of the Basilica image are shown in Fig. 7. The blurred image in Fig. 7(a) was deblurred using the proposed BID scheme. The deblurred image and the corresponding estimated PSF are shown in Fig.

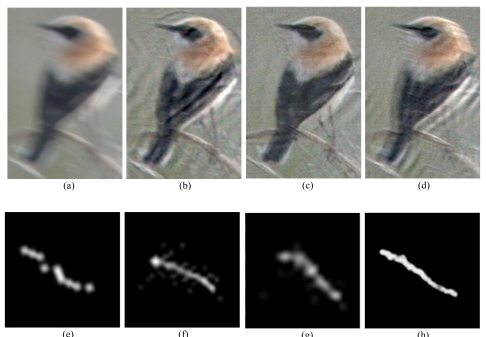

Fig. 6 Deblurring result for Ian-1 image blurred by arbitrary PSF resulting from camera handshake (a) Blurred image. Deblurred using (b) Proposed BID scheme (c) Fergus et al. scheme (d) Whyte et al. scheme with their respective estimated PSFs in (e), (f) and (g).

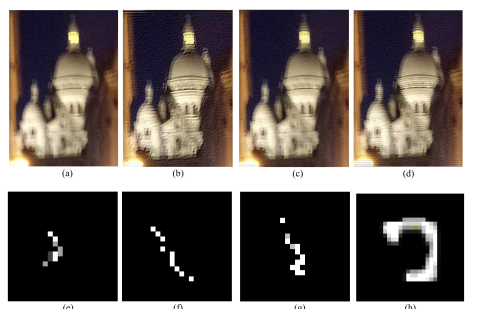

Fig. 7 Deblurring result for Basilica image blurred by arbitrary PSF resulting from camera handshake (a) Blurred image. Deblurred using (b) Proposed BID scheme (c) Fergus et al. scheme (d) Whyte et al. scheme with their respective estimated PSFs in (e), (f) and (g). The hand sketched PSF is shown in $(\mathrm{h})$.

7(b) and Fig. 7(e). The image appears to have recovered well as compared to the deblurred images in Fig. 7(c) and (d) for the BID schemes of Fergus et al. and Whyte et al., respectively. Looking at the estimated PSFs in Fig. 7(f) and Fig. 7(g), it can be seen the estimated PSFs are approximated for few points as compared to the estimated PSF using the proposed scheme in Fig. 7(e). Fig. 8 shows the deblurring results for the Eiffel image.

Fig. 9 shows the deblurring results for the Monument image. The blurred image in Fig. 9 (a) was deblurred using the proposed scheme, Fergus et al. and Whyte et al. scheme in Fig. 9(b), (c) and (d) with their respective estimated PSFs shown in Fig. 9 (e), (f) and (g). In Fig. 9, the image seems to have recovered well, but looking it in detail, the text in the image is unreadable, as shown in Fig. 10.

None of the BID schemes was able to estimate the PSF of the real-life blurred image perfectly. For the proposed BID scheme, the rationale behind its inefficiency can be related to the lack of more extreme image and PSF constraints. However, even in the case of the deblurring schemes of Fergus et al. and Whyte et al., much more advanced image constraints and inplane/out-plane PSF constraints fail to enhance the de- 
blurring performance. This is probably due to blurring models and image/blur statistics constraints that leave large uncertainties in the modeling and computation of the blurring kernel $[2,4]$. Also, the presence of noise in the blurred images may affect the deblurring outcome.

\subsection{SOTA Real Blur Dataset Results}

In order to gauge the proposed scheme's efficiency, it was tested on real-life blurred images. The set of 118 real-life blurred images (with 100 images from Lai dataset and 8 real-blurred images from our dataset) was utilised. These images are blurred from real-life complex-shaped motion blur.

Six of the latest and robust non-reference (totally blind) image quality measures have been used to gauge the performance of the deblurring schemes. These quality measures are based on the Human Visual System (HVS). This includes BRISQUE, NIQE, BLIINDS, DIIVINE, FRIQUEE, SSEQ [34]. Spearman correlation coefficients of the IQM scores with Human scores from various subjects are also presented.

The schemes of Fergus and Whyte were utilised for two reasons: (1) They surpassed the current schemes, particularly for real-life blurred images. (2) Two other methods, Qi Shan and Hirsch et al. performed well but as per our previous study, these two have been outdated.

The following works (with author names) were used as benchmark for comparison of deblurring results in the case of real-life blurred images.

Kupyn (DeblurGAN) [35], Tao (Deep Image Deblurring) [36], Nah (CNN) [37], Mai (Kernel Fusion) [38], Kupyn (DeblurGAN2) [39] and Ramakrishnan (DeepGAN) [40].

Most of the schemes referred above are learningbased and all of them are aimed at handling complexshaped motion blurs. Apart from the proposed scheme, DeblurGAN and Taos Deep Image Deblurring aim at the recovery of artificial as well as real blurred images. Such learning-based BID schemes take a lot of time depending upon the amount of image data used for training e.g. DeblurGAN depicts a training period of six days for $128 \times 128$ image size and around 1000 images while its deblurring algorithm takes only 0.8 seconds.

Using each code along with its pre-trained model, we tested the images for deblurring. Results for testing deblurring of real-life blurred images are presented below. The quality scores are averaged for each quality measure over the set of 118 real-life blurred images.

These quality measures are based on the Human Visual System (HVS). The score for some IQMs were

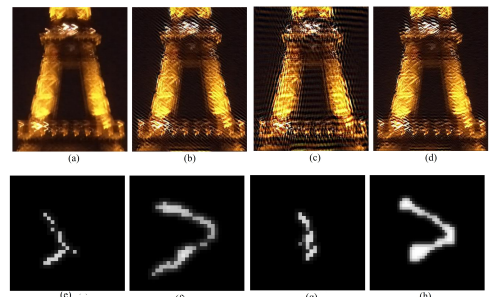

Fig. 8 Deblurring result for Eiffel image blurred by arbitrary PSF resulting from camera handshake (a) Blurred image. Deblurred using (b) Proposed BID scheme (c) Fergus et al. scheme (d) Whyte et al. scheme with their respective estimated PSFs in (e), (f) and (g). The hand sketched PSF is shown in (h).

100 (best) and 0 (worst). Others IQM scores were reordered so they correlate with this range. Table 2 shows the scores.

The proposed scheme is occasionally surpassed by DeblurGAN2 and is nearly matched in performance by DeblurGAN. In our study, we computed the Spearman correlation coefficient of the IQM scores with Human scores from various subjects. The correlation coefficient depicts that the even though the quality measure values of the proposed scheme may be relatively low, they are well aligned with human scores concurring that the restoration results of the proposed scheme are visually better as compared to the benchmarks schemes

The performance of the benchmark schemes, especially training based ones, is severely marred when reallife blurred images are provided. For comparison, the same image size and computing system were used for all testing. We compared our scheme with theirs as they suggest they surpass other previous benchmarks. The proposed scheme's results for both artificial and real deblur outdo the benchmark schemes qualitatively, quantitatively and can handle any type of blur.

\section{Discussion and Analysis}

A novel BID scheme based on GA and utilizing the blind BRISQUE measure as the fitness function is presented for deblurring images corrupted by arbitrarily shaped PSFs. The proposed BID scheme can estimate any parametric or non-parametric PSF coefficients values using an IQM as feedback of deblurred image quality to the BID scheme. During each iteration, a coefficient value is updated in the direction of improved deblurred image quality measured using the blind IQM. The process can be evaluated for a fixed number of iterations or it can be terminated when the difference in the measure value in subsequent iterations is lower than a specified threshold value. 
Table 2 Comparison of blind image quality scores for the proposed scheme vs. the benchmark schemes.

\begin{tabular}{cccccccc}
\hline IQM & $\begin{array}{c}\text { Proposed } \\
\text { Scheme }\end{array}$ & $\begin{array}{c}\text { Kupyn - } \\
\text { Deblur } \\
\text { GAN }\end{array}$ & $\begin{array}{c}\text { Tao - Deep } \\
\text { Image } \\
\text { Deblurring }\end{array}$ & $\begin{array}{c}\text { Nah- } \\
\text { CNN }\end{array}$ & $\begin{array}{c}\text { Mai - } \\
\text { Kernel } \\
\text { Fusion }\end{array}$ & $\begin{array}{c}\text { Kupyn - } \\
\text { Deblur } \\
\text { GAN2 }\end{array}$ & $\begin{array}{c}\text { Ramakrishnan - } \\
\text { DeepGAN }\end{array}$ \\
\hline BRISQUE & 67.51 & 59.71 & 46.46 & 62.49 & 65.50 & 66.00 & 61.95 \\
BLIINDS & 66.48 & 54.92 & 52.88 & 57.79 & 59.85 & 57.53 & 62.02 \\
DIIVINE & 63.57 & 52.05 & 49.69 & 54.98 & 56.80 & 54.45 & 59.02 \\
FRIQUEE & 63.38 & 55.57 & 42.56 & 58.46 & 60.95 & 62.01 & 57.89 \\
ILNIQE & 54.58 & 46.81 & 48.29 & 45.68 & 36.66 & 56.22 & 50.88 \\
SSEQ & 79.91 & 67.40 & 68.89 & 69.40 & 71.52 & 72.78 & 73.32 \\
\hline Mean Score & 65.91 & 56.08 & 51.46 & 58.13 & 58.55 & 61.50 & 60.85 \\
\hline Spearman Corr Coeff & 0.0868 & -0.1747 & 0.1845 & -0.1426 & -0.1634 & 0.1112 & -0.1252 \\
\hline
\end{tabular}

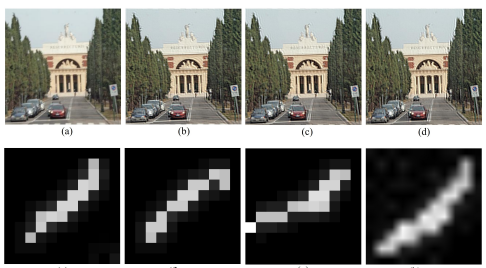

Fig. 9 Deblurring result for Monument image blurred by arbitrary PSF resulting from camera handshake (a) Blurred image. Deblurred using (b) Proposed BID scheme (c) Fergus et al. scheme (d) Whyte et al. scheme with their respective estimated PSFs in (e), (f) and (g).
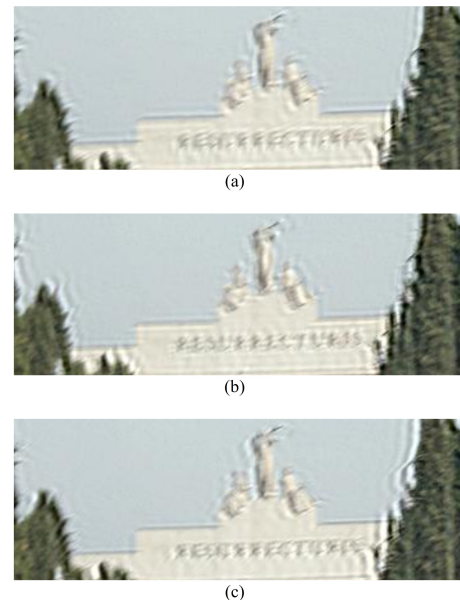

Fig. 10 Deblurring results for section of Monument image using (a) Proposed BID scheme (b) Fergus et al. scheme and (c) Whyte et al. scheme. The text in the image is unreadable in all cases.

The proposed BID schemes estimated PSF coefficients are not exactly the same as the original PSF but are rather a near approximation of the original blurring PSF. This affects the brightness and contrast of the deblurred images thus rendering the full-reference IQMs PSNR, MSSIM, UQI etc invalid for quantitative image quality evaluation. Hence, non-reference IQMs
BRISQUE and NIQE were used for computation of deblurred image quality.

Initially, a self-coded GA variant was employed for optimizing the search algorithm which was later replaced by MATLAB based GA in its optimization toolbox. The proposed BID was successfully evaluated in parallel on a multiple-core machine. The proposed BID scheme requires a fixed PSF size input from the user. In this regard, a method for visual judgment based PSF size estimation prior to deblurring is discussed in Section IV. In the case of real blurred images, the PSF size was estimated by inspecting a blur region in the image.

Since the BID scheme can also be extended for uniform parametric blurs, the algorithm was first evaluated for the less complex parametric form blurs before testing it for arbitrarily shaped PSFs. Results presented here were obtained when the algorithm stopped, as the tolerance value for the fitness function was attained. The restored images appear sharper and much more detailed than its blurred counterpart.

Real-life blurred images were used to test the efficacy of the deblurring scheme for practical application. The images depict motion blur resulting from camera handshake. The proposed BID scheme has been compared with other deblurring schemes that include the single image motion deblurring scheme of Fergus et al. [3] and the single/noisy-paired motion deblurring scheme of Whyte et al. [5]. None of the BID schemes was able to estimate the PSF of the real-life blurred image perfectly.

For the proposed BID scheme, the rationale behind its inefficiency can be related to the lack of more extreme image and PSF constraints. However, even in the case of the deblurring schemes of Fergus et al. and Whyte et al., much more advanced image constraints and in-plane/out-plane PSF constraints fail to enhance the deblurring performance. This is probably due to blurring models and image/blur statistics constraints that leave large uncertainties in the modelling and com- 
putation of the blurring kernel. Also, the presence of noise in the blurred images may affect the deblurring outcome.

\section{Conclusions}

The problem of deblurring of images corrupted by complex motion blurs has been analyzed and a BID scheme is proposed. The proposed BID can handle both parametric and arbitrarily shaped PSF estimation using a single algorithm, for single-shot blurred images, with enhanced optimization through GA. The deblurring results show effective PSF estimation capability for parametric and arbitrarily shaped PSFs in the case of artificially blurred images. For real-life blurred images resulting from camera handshake, the proposed scheme supasses the benchmark schemes and estimates the blurring kernel well. Different IQMs and restoration filters can be easily incorporated in the proposed BID in order to investigate their performance especially in enhancing the deblurred image quality. Modeling and deblurring the complex camera handshake PSFs effectively still remains a challenging task particularly for real-life blurred images.

\section{Conflict of interest}

The authors declare that they have no conflict of interest.

\section{References}

1. J. Biemond, R. L. Lagendijk, and R. M. Mersereau, "Iterative methods for image deblurring," Proceedings of the IEEE, vol. 78, no. 5, pp. 856-883, 1990.

2. M. S. C. Almeida and L. B. Almeida, "Blind and semiblind deblurring of natural images," IEEE Transactions on Image Processing, vol. 19, no. 1, pp. 36-52, 2010.

3. R. Fergus, B. Singh, A. Hertzmann, S. T. Roweis, and W. T. Freeman, "Removing camera shake from a single photograph," ACM Transactions on Graphics, vol. 25, no. 3, pp. 787-794, 2006.

4. Q. Shan, J. Jia, and A. Agarwala, "High-quality motion deblurring from a single image," ACM Transactions on Graphics, vol. 27, no. 3, p. 10, 2008.

5. O. Whyte, J. Sivic, A. Zisserman, and J. Ponce, "Nonuniform deblurring for shaken images," International Journal of Computer Vision, vol. 98, no. 2, pp. 168-186, 2012.

6. O. Whyte, J. Sivic, and A. Zisserman, "Deblurring shaken and partially saturated images," 2011.

7. M. Hirsch, C. J. Schuler, S. Harmeling, and B. Scholkopf, "Fast removal of non-uniform camera shake," 2011.

8. A. Gupta, N. Joshi, C. L. Zitnick, M. Cohen, and B. Curless, "Single image deblurring using motion density functions," 2010.
9. H. E. Fortunato and M. M. Oliveira, "Fast high-quality non-blind deconvolution using sparse adaptive priors," The Visual Computer, vol. 30, no. 6-8, pp. 661-671, 2014.

10. A. Bini and M. Bhat, "A nonlinear level set model for image deblurring and denoising," The Visual Computer, vol. 30, no. 3, pp. 311-325, 2014.

11. Q. Feng, H. Fei, and W. Wencheng, "Blind image deblurring with reinforced use of edges," The Visual Computer, vol. 35, no. 6-8, pp. 1081-1090, 2019.

12. X. Zhang, F. Sun, G. Liu, and Y. Ma, "Non-blind deblurring of structured images with geometric deformation," The Visual Computer, vol. 31, no. 2, pp. 131-140, 2015.

13. R. Tubbs, Lucky Exposures: Diffraction Limited Astronomical Imaging through the Atmosphere. Phd dissertation, 2003.

14. A. Khan and H. Yin, "Efficient blind image deconvolution using spectral non-gaussianity," Integrated ComputerAided Engineering, vol. 19, no. 4, pp. 331-340, 2012.

15. I. Hussain, Non-Gaussianity Based Image Deblurring and Denoising. Thesis, 2008.

16. A. Hyvarinen, "Fast and robust fixed-point algorithms for independent component analysis," IEEE Transactions on Neural Networks, vol. 10, no. 3, pp. 626-634, 1999.

17. A. Hyvarinen and E. Oja, "Independent component analysis: Algorithms and applications," Neural Networks, vol. 13 , no. 4-5, pp. 411-430, 2000.

18. A. Khan and H. Yin, "Spectral non-gaussianity for blind image deblurring," 2011.

19. A. Mittal, A. K. Moorthy, and A. C. Bovik, "Noreference image quality assessment in the spatial domain," IEEE Transactions on Image Processing, vol. 21, no. 12 , pp. 4695-4708, 2012.

20. A. Mittal, R. Soundararajan, and A. C. Bovik, "Making a "completely blind" image quality analyzer," IEEE Signal Processing Letters, vol. 20, no. 3, pp. 209-212, 2013.

21. H. J. Yin and I. Hussain, "Independent component analysis and non-gaussianity for blind image deconvolution and deblurring," Integrated Computer-Aided Engineering, vol. 15, no. 3, pp. 219-228, 2008.

22. A. Khan and H. Yin, "Quality measures for blind image deblurring," 16-17 July 20122012.

23. A. Mittal, A. K. Moorthy, and A. C. Bovik, "Brisque software release," 2011.

24. A. Mittal, A. K. Moorthy, and A. C. Bovik, "Niqe software release," 2012.

25. "Desktop nexus wallpaper image database."

26. "Matlab optimization toolbox."

27. W. Lai, J. Huang, Z. Hu, N. Ahuja, and M. Yang, "A comparative study for single image blind deblurring," in 2016 IEEE Conference on Computer Vision and Pattern Recognition (CVPR), pp. 1701-1709, June 2016.

28. D. Brunet, E. R. Vrscay, and Z. Wang, "On the mathematical properties of the structural similarity index," IEEE Transactions on Image Processing, vol. 21, no. 4, pp. 1488-1499, 2012.

29. Z. Wang, A. C. Bovik, H. R. Sheikh, and E. P. Simoncelli, "Image quality assessment: From error visibility to structural similarity," IEEE Transactions on Image Processing, vol. 13, no. 4, pp. 600-612, 2004.

30. Z. Wang and Q. Li, "Information content weighting for perceptual image quality assessment," IEEE Transactions on Image Processing, vol. 20, no. 5, pp. 1185-1198, 2011.

31. Z. Wang and A. C. Bovik, "A universal image quality index," IEEE Signal Processing Letters, vol. 9, no. 3, pp. 81-84, 2002. 
32. A. Khan, Efficient Methodologies for Single-Image Blind Deconvolution and Deblurring. Phd dissertation, 2014.

33. R. Fergus, B. Singh, A. Hertzmann, S. T. Roweis, and W. T. Freeman, "Blurred images for blind image deblurring."

34. "Live non-reference image quality database."

35. O. Kupyn, V. Budzan, M. Mykhailych, D. Mishkin, and J. Matas, "Deblurgan: Blind motion deblurring using conditional adversarial networks," in Proceedings of the IEEE conference on computer vision and pattern recognition, pp. 8183-8192, 2018.

36. X. Tao, H. Gao, X. Shen, J. Wang, and J. Jia, "Scalerecurrent network for deep image deblurring," in Proceedings of the IEEE Conference on Computer Vision and Pattern Recognition, pp. 8174-8182, 2018.

37. S. Nah, T. Hyun Kim, and K. Mu Lee, "Deep multi-scale convolutional neural network for dynamic scene deblurring," in Proceedings of the IEEE Conference on Computer Vision and Pattern Recognition, pp. 3883-3891, 2017.

38. L. Mai and F. Liu, "Kernel fusion for better image deblurring," in Proceedings of the IEEE Conference on Computer Vision and Pattern Recognition, pp. 371-380, 2015.

39. O. Kupyn, T. Martyniuk, J. Wu, and Z. Wang, "Deblurgan-v2: Deblurring (orders-of-magnitude) faster and better," in Proceedings of the IEEE International Conference on Computer Vision, pp. 8878-8887, 2019.

40. S. Ramakrishnan, S. Pachori, A. Gangopadhyay, and S. Raman, "Deep generative filter for motion deblurring," in Proceedings of the IEEE International Conference on Computer Vision Workshops, pp. 2993-3000, 2017. 